\title{
The Initial Months of Antiretroviral Therapy and Its Influence on AGEs, HMGB1, and sRAGE Levels in Asymptomatic HIV-Infected Individuals
}

\author{
Karen Ingrid Tasca, ${ }^{1}$ Juliana Trindade Caleffi, ${ }^{1}$ Camila Renata Correa, ${ }^{2}$ \\ Mariana Gatto, ${ }^{1}$ Caio Cavassan de Camargo, ${ }^{1}$ Monica Bannwart Mendes, ${ }^{1}$ \\ Marjorie de Assis Golim, ${ }^{3}$ Mara Biasin, ${ }^{4}$ and Lenice do Rosário de Souza ${ }^{1}$ \\ ${ }^{1}$ Department of Tropical Diseases, Botucatu Medical School (FMB), Universidade Estadual Paulista (UNESP), Botucatu, SP, Brazil \\ ${ }^{2}$ Department of Pathology, FMB/UNESP, Botucatu, SP, Brazil \\ ${ }^{3}$ Hemocenter, FMB/UNESP, Botucatu, SP, Brazil \\ ${ }^{4}$ Department of Biomedical and Clinical Sciences, University of Milan, Milan, Italy
}

Correspondence should be addressed to Karen Ingrid Tasca; karenitasca@hotmail.com

Received 8 July 2016; Revised 4 October 2016; Accepted 26 October 2016

Academic Editor: Gabriella D’Ettorre

Copyright (c) 2016 Karen Ingrid Tasca et al. This is an open access article distributed under the Creative Commons Attribution License, which permits unrestricted use, distribution, and reproduction in any medium, provided the original work is properly cited.

\begin{abstract}
The development of the typical comorbidities of aging which currently affects people living with HIV/AIDS (PLWHA) can be partially ascribed to the persistent immune activation and chronic inflammation characterizing these individuals. The aim of this study was to analyze the effect exerted by combined antiretroviral therapy (cART) administration on plasma levels of HMGB1 (high mobility group box protein-1), AGEs (advanced glycation end products), their soluble receptor sRAGE, cytokines, Creactive protein (CRP), and some metabolic markers in asymptomatic PLWHA. Analyses were performed longitudinally in 30 PLWHA, before and about 6-12 months after cART initiation. We observed that lower levels of AGEs in post-cART group were accompanied by an increase of CRP and triglyceride levels already in the early months of therapy. Because of the current ever-earlier recommendations to start CART and its prolonged use, these and other markers should be investigated in order to monitor and postpone the appearance of non-AIDS comorbidities in PLWHA.
\end{abstract}

\section{Introduction}

Although the HIV epidemics affect more than 36 million individuals worldwide, with a global prevalence of almost $1 \%$ [1], life expectancy of people living with HIV/AIDS (PLWHA) is very similar to that of the general uninfected population, which makes the clinical approach to the infection similar to that of other chronic diseases that characterize aging [1-3]. This improvement is due to the combination of several actions and advances in this area, especially the guaranteed access to potent and combined antiretroviral therapy (cART) to the majority of the population $[1,2,4]$.

However, the great challenge today is to delay the onset and severity of non-AIDS-defining comorbidities, which appear much earlier in HIV-infected individuals [5] and include cardiovascular diseases, diabetes mellitus, kidney, liver, and bone alterations, and neoplasia [4-8]. The onset and severity of these comorbidities are strictly dependent on the intense immune activation and chronic inflammation affecting PLWHA, regardless of cART administration, which can only partly reduce these conditions and the derived products $[3,7]$. Furthermore, the toxicity and adverse effects of cART can also foster the development of such diseases [7, 9], mainly dyslipidemia.

Advanced glycation end products (AGEs) are a consequence of oxidative stress which occur during normal physiological conditions such as aging. However, if they are not excreted in the urine or their formation is exacerbated as in the case of diabetes hyperglycemia and other diseases, they accumulate in the vascular tissue, causing harmful 
effects $[10,11]$. Furthermore, their binding to their receptors (RAGE) promotes the activation of proinflammatory and procoagulant cellular pathways, thus increasing the production of adhesion molecules, cytokines such as IL- 6 and TNF$\alpha$, and the generation of nitric oxide (NO). These mechanisms can influence the course of HIV infection and contribute to a number of serious pathological conditions, including the development of atherosclerosis, diabetes mellitus, osteoporosis, neoplasia, neurological disorders, among others [11-17].

It has also been observed that an alternative mRNA splicing which removes RAGE transmembrane domain [18, 19] results in the production of a soluble RAGE (sRAGE) [20]. sRAGE competes with the transmembrane receptor sequestering its ligands and preventing the activation of proinflammatory pathways [19]. Therefore, changed sRAGE levels have been associated with increased cardiovascular risk [14, 21], hypercholesterolemia [22], and high blood pressure [23], as well as diabetes mellitus [10], and new drugs prompting RAGE blocking and/or sRAGE increase have been proposed for future therapies [18].

High mobility group box protein-1 (HMGB1) is another key factor in promoting inflammation. It can be actively secreted following different stimuli, such as cytokines, endotoxins, and pathogen-associated molecular patterns (PAMPs) [24], or, passively, by necrotic cells [25], common conditions observed in HIV-individuals. In the extracellular environment, HMGB1 activates various pattern-recognition receptors (PRRs), such as RAGE and toll-like receptors (TLR) [2628], and acts as a proinflammatory cytokine [25] and alarmin, stimulating both innate and adaptive immune system to prevent damage and promoting regeneration and repair [29]. In humans, its high systemic levels are related to several acute and chronic inflammatory conditions, including pancreatitis, diabetes mellitus, rheumatologic and autoimmune diseases, and cancer [17, 28, 30-32].

Few studies have investigated the role of these markers in HIV infection and how their expression is altered following cART initiation. Considering the need for better understanding of inflammatory mechanisms and cellular activation in this patients, as well as their influence on the "premature aging" phenomenon, the aim of this study was to compare the levels of HMGB1, AGEs, sRAGE, and some inflammatory biomarkers in the plasma of asymptomatic PLWHA before cART and approximately six months to one year after its introduction.

\section{Materials and Methods}

2.1. Study Design. This is a longitudinal study involving 30 HIV-infected subjects, attended at the Specialist Outpatient Service for Infectious Diseases "Domingos Alves Meira" (SAEI-DM), at the Botucatu Medical School Complex (FMB)-UNESP, in São Paulo state, Brazil. This service covers about 600 people from Botucatu city and surrounding area and, for this study, 150 people (those cART-naïve) were interviewed, with only 30 of them being included after applying the exclusion criteria. The collection of data and biological samples was carried out in two stages, before therapy initiation (M0) and approximately six to twelve months after this intervention (M1), according to the patient regular return in the service. This research project was approved by the local Research Ethics Committee and was conducted between the years 2012 and 2015.

2.2. Inclusion and Exclusion Criteria. The inclusion criteria were age between 20 and 50 years, no previous cART administration, and signing an informed consent form.

Patients excluded were those with any of the following conditions: use of vitamin supplements, cancer history (current or previous), anorexia, morbid obesity, diabetes mellitus, cardiovascular, genetic, or autoimmune diseases, organ transplants, pregnancy at any stage or breastfeeding, coinfections such as tuberculosis, chronic viral hepatitis, cerebral or disseminated toxoplasmosis, and use of illicit drugs and alcohol. For the following criteria, exclusion occurred when patients concomitantly reported two of them or more: regular performance of intense physical exercise; use of antibiotics, anxiolytics, or antidepressants; active smoking. People with only one of these conditions were included because the statistical analysis was conducted in adjusted models for these variables.

2.3. Sociodemographic and Clinical Data. Sociodemographic and clinical data were collected by interviews and from the patients' medical records. Most of the participants were males $(60.0 \%)$ aged approximately 34 years $( \pm 8.2)$, heterosexual $(66.6 \%)$, and single $(36.6 \%)$. The mean duration of HIV infection, considering its diagnosis, was 2.2 years $( \pm 3.2)$, and $70 \%$ of subjects showed a CD4+ T cells count lower than 500 cells $/ \mathrm{mm}^{3}$ in the first collection, with nadir of $335.7 \pm 211.3$ cells $/ \mathrm{mm}^{3}$.

Furthermore, $30.0 \%$ were active smokers, $23.3 \%$ practiced intense physical activity, and $6.6 \%$ were taking anxiolytics and/or antidepressants.

Results from the following tests were collected from the medical records: plasma HIV viral load (VL), CD4+ T cells count, glucose, triglycerides, total cholesterol, and C-reactive protein (CRP). Adherence to cART was considered, at first, based on the reports of patients and subsequently verified with the records of medicine withdrawal, at the pharmacy service.

2.4. Performance of Laboratory Tests. Twelve milliliters of blood were collected into an EDTA containing tube from each patient included in the study. The sample was maintained in a cooled and dark environment for 2-3 hours and then centrifuged at 1,500 rpm (Centrifuge 5702R Eppendorf) for 10 minutes at $10^{\circ} \mathrm{C}$. Six plasma aliquots per individual were stored at $-80^{\circ} \mathrm{C}$ until the tests were performed.

2.4.1. Measurement of Plasma HMGB1. A sandwich-type immunoenzymatic assay (ELISA) was performed, using $100 \mu \mathrm{L}$ of diluted sample $(1: 10)$ and following the manufacturer's specifications from commercial kit (MyBioSource, item MBS2707497). Plasma HMGB1 concentration was determined by spectrophotometry, at $450 \mathrm{~nm}$. Results were expressed as $\mathrm{pg} / \mathrm{mL}$ using the optical density (OD) of the curves and samples for this calculation. 
TABLE 1: Clinical data of 30 PLWHA before and after cART initiation.

\begin{tabular}{lccc}
\hline Variable & M0 (MEAN \pm SD) & M1 (MEAN \pm SD) & $p$ value \\
\hline CD4+ T count $\left(\right.$ cells $\left./ \mathrm{mm}^{3}\right)$ & $454.69 \pm 136.38$ & $625.07 \pm 276.94$ & $<0.0001$ \\
HIV VL $\left(\right.$ RNA copies $\left./ \mathrm{mm}^{3}\right)$ & $126,550.8 \pm 9,756.2$ & $30,813.3 \pm 27,463.9$ & 0.019 \\
Undetectable HIV VL & - & $80.0 \%$ & - \\
cART regimen - NRTI + NNRTI & - & $83.3 \%$ & - \\
cART regimen - NRTI + PI/r & - & $16.7 \%$ & - \\
\hline
\end{tabular}

PLWHA: people living with HIV/AIDS; cART: combined antiretroviral therapy; VL: viral load; NRTI: nucleoside reverse transcriptase inhibitor; NNRTI: nonnucleoside reverse transcriptase inhibitor; PI/r: protease inhibitors reinforced with ritonavir; M0: before cART initiation; M1: after cART initiation. Statistical tests: gamma distribution for CD4+ T cell count and Poisson to HIV VL.

2.4.2. Measurement of Plasma AGEs. A commercial ELISA kit (Cell Biolabs Inc., item STA-317) was used following the protocol provided by the manufacturer, with $100 \mu \mathrm{L}$ of pure sample. Absorbance was read by a spectrophotometer at a wavelength of $450 \mathrm{~nm}$ and, based on the OD of the sample and curve, AGEs levels were calculated in $\mu \mathrm{g} / \mathrm{mL}$.

2.4.3. Measurement of sRAGE. Using $100 \mu \mathrm{L}$ of pure samples, the sandwich ELISA protocol was developed according to the manufacturer's instructions (R\&D Systems, item DRG00). Reading was performed immediately by a spectrophotometer at the wavelength of $450 \mathrm{~nm}$. OD of the samples and curve were calculated and expressed in $\mathrm{pg} / \mathrm{mL}$.

2.4.4. Measurement of IL-6, IL-8, and IL-10. Cytokine measurement was performed by flow cytometry (FACSCaliburE34297502374) using the CBA (cytometric bead array) kit of inflammatory cytokines, according to the manufacturer's instruction manual (BD-Becton, Dickinson and Company, item 551811). The fluorescence intensity, from the cytometer, showed the amount of cytokines present in the sample. With the samples, standards containing known concentrations of each cytokine were analyzed together for the drawing of the curve, reading, and establishment of the respective detection limits. Results were obtained in $\mathrm{pg} / \mathrm{mL}$ by the software FCAP $\operatorname{array}^{\mathrm{TM}}$ (BD-Becton, Dickinson and Company).

2.5. Analysis of Results. Continuous variables were expressed as mean (ME) and standard deviation (SD) and categorical variables by frequency and percentage (\%). We used Poisson test or gamma distribution for nonparametric variables and negative binomial or one-way ANOVA with subsequent Tukey-Kramer post hoc test for those parametric data. We also performed Pearson correlations among the continuous clinical variables: AGE, sRAGE, HMGB1, glucose, triglycerides, total cholesterol, CRP, cytokines, and CD4+ T cells.

All calculations were adjusted for age, sex, and tobacco use, practice of intense physical activity, and use of anxiolytics and/or antidepressants.

Significant differences were considered when $p$ values were less than or equal to 0.05 . All analyses were performed with help from professionals at the institution's Research Support Office, using SAS for Windows, version 9.2.

\section{Results}

In this study, we observed an increase in the patients' CD4+ $\mathrm{T}$ counts $\left(454.69 \pm 136.38\right.$ versus $625.07 \pm 276.94 \mathrm{cells} / \mathrm{mm}^{3}$, $p<0.0001$ ) after cART (Table 1). It is noteworthy that the average of CD4+ T cells gain was 170.38 cells $/ \mathrm{mm}^{3}$ and it was higher in individuals with a count lower than 500 cells $/ \mathrm{mm}^{3}$ (209.71 cells $\left./ \mathrm{mm}^{3}\right)$ at the beginning of the therapy, compared to those with an initial count above 500 cells $/ \mathrm{mm}^{3}$ (109.62 cells $/ \mathrm{mm}^{3}$ ). In Brazil, although the treatment is offered to all HIV-infected, before December 2013, therapy was initiated only for symptomatic patients or patients with $\mathrm{CD} 4+\mathrm{T}$ counts below 500 cells $/ \mathrm{mL}$.

Among the PLWHA studied, 83.3\% were under a therapeutic regimen consisting of the nonnucleoside reverse transcriptase inhibitors (NNRTI), and $80.0 \%$ had undetectable $\mathrm{VL}$ at M1. At the moment of this second collection, the participants were asked about adherence to therapy, which was reported by $86.6 \%$ of them. The average of VL measurements in pre-cART was $126,550.8( \pm 69,756.2) \mathrm{RNA}$ copies $/ \mathrm{mm}^{3}$. At M1, $80.0 \%$ of the patients had undetectable VL; in the remaining $20 \%$ patients, VL was $30,813.3( \pm 27,463.9)$ RNA copies $/ \mathrm{mm}^{3}$. Viral load differences between M0 and M1 reached statistical significance $(p=0.019)$ (Table 1$)$.

Differences in triglyceride serum levels were observed as well $(142.90 \pm 53.38$ versus $184.90 \pm 115.07 \mathrm{mg} / \mathrm{dL}, p=0.001)$ comparing $\mathrm{M} 0$ and $\mathrm{M} 1$. Conversely, no differences in glucose and total cholesterol were observed at the two moments (Figure 1).

After cART introduction, AGEs plasma levels $(0.64 \pm 0.12$ versus $0.49 \pm 0.10 \mathrm{pg} / \mathrm{mL}, p<0.001$ ) decreased significantly (Figure 2). In order to investigate the influence of CD4+ T cells on this change, patients were divided into two groups according to their CD4 T counts at M0 (> or $<$ than 500 CD4 cells $/ \mathrm{mm}^{3}, n=11$ and 19 individuals, resp.). Results suggest that AGEs decrease occurred only in individuals with an initial CD $4+\mathrm{T}$ count below 500 cells $/ \mathrm{mm}^{3}(p=0.049)$. There were no differences for sRAGE ( $p=0.175)$ or HMGB1 $(p=0.119)$ as shown in Figure 2.

CRP levels also increased after six months of cART ( $0.74 \pm$ 0.41 versus $1.06 \pm 0.99 \mathrm{mg} / \mathrm{dL}, p=0.006$ ); however, no differences in cytokine levels were observed comparing M0 and M1 (Figure 3). 


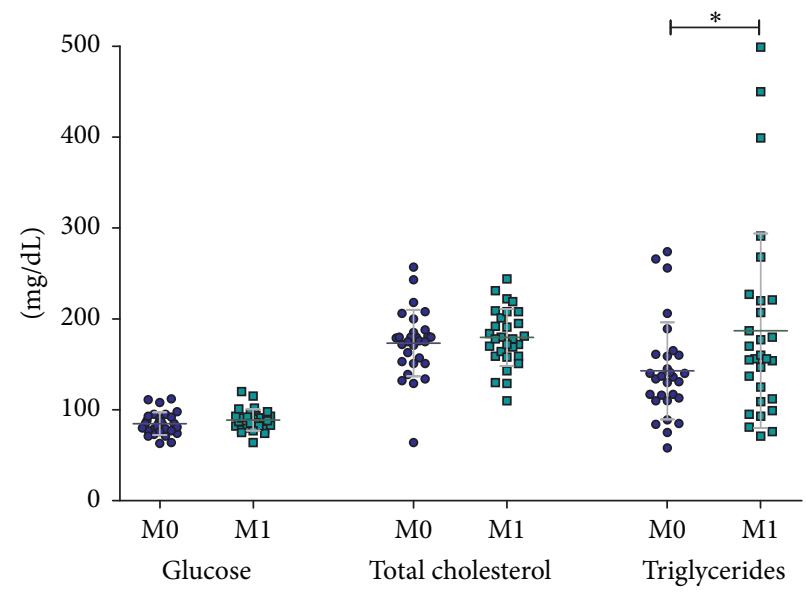

FIGURE 1: Mean of blood glucose measurements, total cholesterol, and triglycerides of 30 PLWHA, before and after cART initiation. PLWHA: people living with HIV/AIDS; cART: combined antiretroviral therapy; M0: before cART initiation; M1: after cART initiation. Statistical tests: gamma distribution for triglycerides and ANOVA with post hoc Tukey's tests for the other variables. ${ }^{*} p<0.001$.
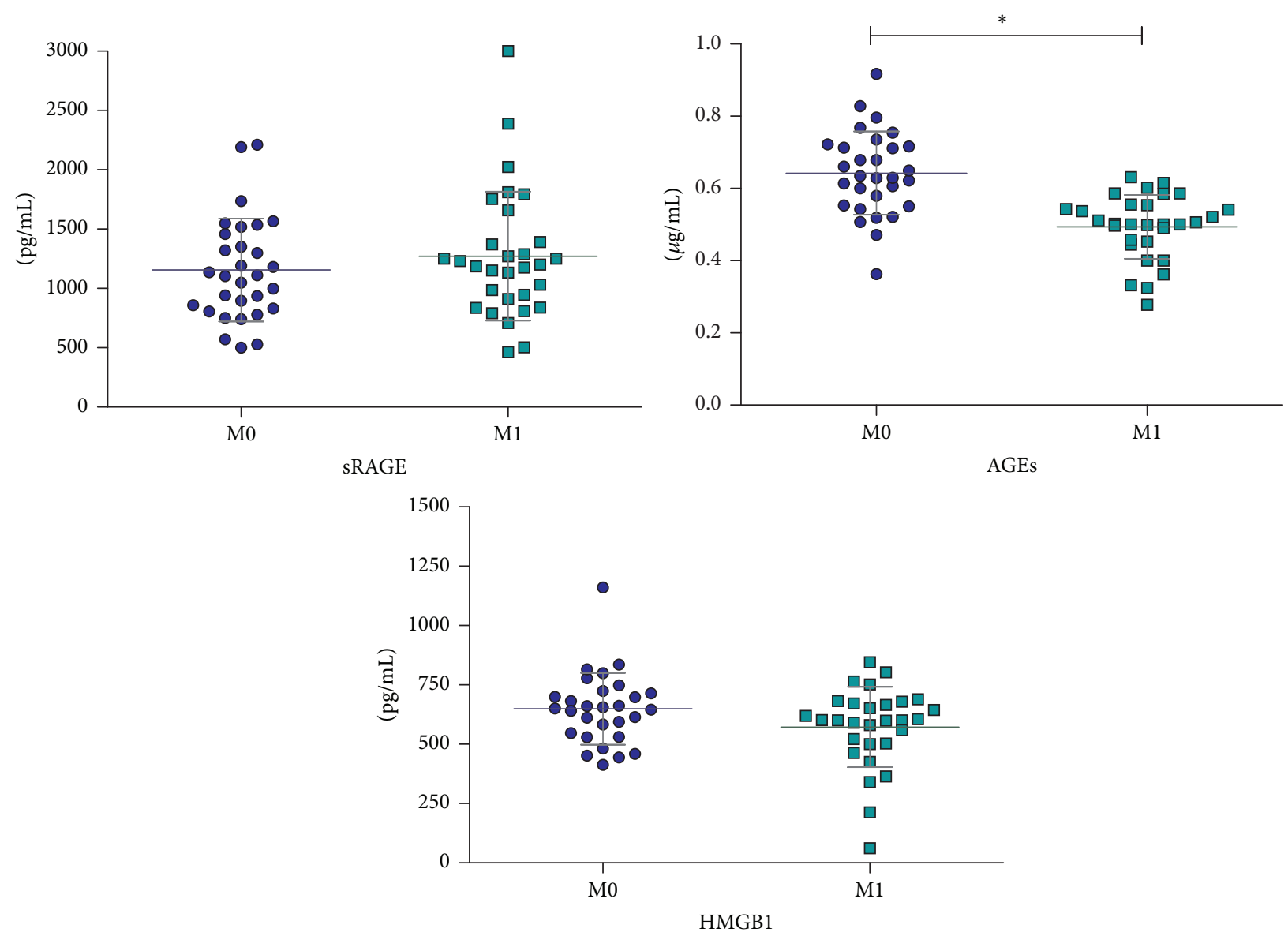

Figure 2: Plasma levels of RAGEs, AGEs, and HMGB1 of 30 PLWHA, before and after cART initiation. PLWHA: people living with HIV/AIDS; cART: combined antiretroviral therapy; AGEs: advanced glycation end products; sRAGE: soluble receptor of AGEs; HMGB1: high mobility group box protein-1. M0: before cART initiation; M1: after cART initiation. Statistical tests: ANOVA with post hoc Tukey's tests. ${ }^{*} p<0.0001$. 


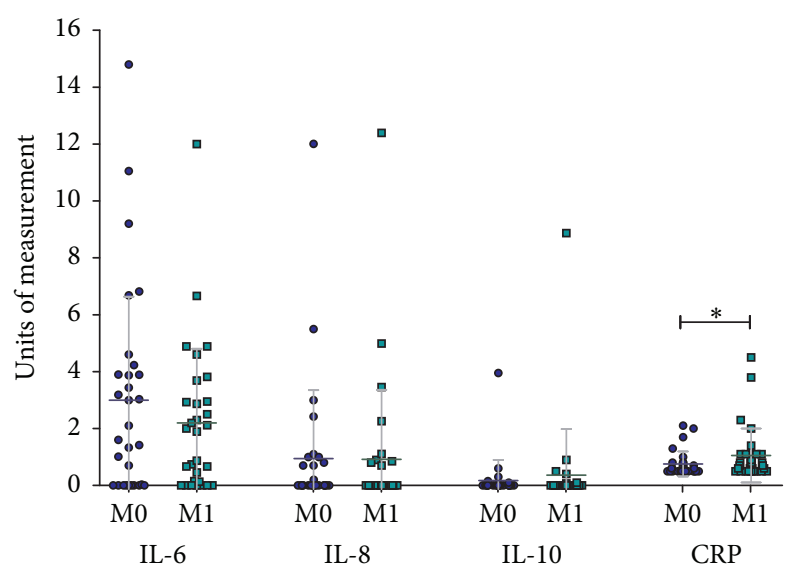

FIGURE 3: Changes in plasma levels of cytokines and CRP of 30 PLWHA, before and after cART initiation. PLWHA: people living with HIV/AIDS; cART: combined antiretroviral therapy; IL: interleukin; CRP: C-reactive protein; M0: before cART initiation; M1: after cART initiation. Statistical tests: gamma distribution. ${ }^{*} p<$ 0.01 . Units of measurement: " $\mathrm{mg} / \mathrm{dL}$ " to CRP and " $\mathrm{pg} / \mathrm{mL}$ " to cytokines.

After the Pearson correlation among the studied variables, we only observed that IL-10 level was positively correlated with IL-8 and CRP and CD4+ T cell count was negatively correlated with IL-6 and IL-8.

\section{Discussion}

The risk of progression to AIDS and death are related to high viremia and low CD4+ T lymphocyte level [33], which reinforces the importance of adherence to cART and regular monitoring of all these parameters in the clinical practice. The majority of treatment-naïve patients who start cART show a decrease in VL to undetectable levels $\left(<40\right.$ copies $\left./ \mathrm{mm}^{3}\right)$ and an increase in $\mathrm{CD} 4+\mathrm{T}$ cell counts after approximately six months of therapy [1]. This trend mirrors the results obtained in this study as well as the actual tendency in Brazil, as shown in the last Epidemiological Bulletin, where undetectable VL is achieved by $78 \%$ of the patients after therapy in both 2013 and 2014 [4].

In order to delay the decrease in CD4+ T cells to very low levels, current recommendations are to begin treatment as soon as the diagnosis of HIV infection is confirmed, regardless of CD4+ T counts. Despite this knowledge, 26\% of Brazilians have a very late diagnosis, when their CD4+ $\mathrm{T}$ count is below 200 cells $/ \mathrm{mm}^{3}$ and, as a result, they also begin treatment later [4]. Likewise, in this study, $70 \%$ of the patients showed fewer than 500 cells $/ \mathrm{mm}^{3}$ and a nadir of $335.7 \mathrm{cells} / \mathrm{mm}^{3}$ at M0. However, similar to a previous study performed by our research group [34], the gain of CD4+ T cells after cART was more evident in patients who had lower counts at M0.

Despite the benefits of cART in the stabilization of HIV infection, there is evidence that the therapy is accompanied by some adverse effects, especially considering its long-term administration $[9,35]$. Although increased oxidative stress [35] and dyslipidemia $[9,36]$ are some of the most evident side effects, in our study, no differences in glucose and total cholesterol levels were observed after therapeutic intervention. However, we cannot exclude that longer treatment could provoke these problems, as Pinto Neto et al. [35] observed that dyslipidemia and glucose disturbances occurred after three years of cART use. On the other hand, in our study, patients showed a significant increase in triglyceride levels already in the first months of treatment, with no influence of the scheme used, protease inhibitors (PI), or NNRTI (data not shown). However, other authors reported that the metabolic imbalance may be associated with different cART regimens, especially those including some PI [36]. Such changes should be monitored, as they contribute to the appearance of comorbidities not related to AIDS, such as cardiovascular disease and diabetes mellitus [8].

Early aging has been associated with and explained by the persistent immune activation and inflammation shown by HIV-infected individuals due to residual HIV replication in lymphoid tissues, the intense microbial translocation that may occur, the metabolic imbalance, and the instability of the cytokine profile and of the redox status, among others [7, 3739]. It is known that cART reduces inflammation but does not restore the physiological base level $[3,7]$. Referring to the inflammatory process, cytokines and CRP measurements were studied here before and after cART introduction. Most of our patients showed undetectable levels of cytokines, which, in a way, could be justified by the asymptomatic condition and absence of comorbidities in the studied time. Furthermore, plasma levels of cytokines, except of TNF- $\alpha$, were also extremely low in study by Fahey [40] also with HIV-infected individuals. Another explanation for these low values is the choice of technique used to measure them (CBA), which may have been less sensitive compared to traditional ELISA.

No significant statistical differences were observed in IL6 , IL-8, and IL-10 levels production here, although IL-6 has presented a slightly lower value and the IL-10 levels were more prevalent after cART initiation. Other authors reported a decrease in IL-6 synthesis after therapy introduction, considering a longer intervention, of one to two years [41], so, this inconsistency may be related to the short period of cART administration in our study. The slight decrease in IL-6 levels can be associated with lower viral load after cART initiation, to their negative correlation with $\mathrm{CD} 4+\mathrm{T}$ cell count, also found in this study, and by the small increase in IL-10 levels. Besides that, we found a positive correlation between IL-10 with IL-8 and CRP, indicating that probably high IL-10 levels would be required to cause reduction in other inflammatory markers.

In the present study, the CRP levels of all patients were normal or slightly high, considering the reference value at the two moments studied; however, a significant increase in its synthesis was observed after treatment. Thus, these results do not seem to suggest a contribution from cART to inflammation decrease during the study period. Actually, our hypothesis is that CRP elevation may be associated with the increase of parameters related to metabolic syndrome, such 
as triglycerides, which in our study also showed higher values after therapy. Similar results were found by Masiá et al. [42] and Guimarães et al. [43] with treated compared to cARTnaïve patients, which demonstrated a positive correlation between CRP or hs-CRP and triglycerides, lipids, insulin levels, and systolic and diastolic blood pressure among other parameters, including Framingham risk score [42, 43]. Neither viral load nor the CD4+ T counts were correlated with CRP in these studies; therefore, CRP and hsCRP may then be markers for cardiovascular risk linked to HIV infection and cART $[42,43]$.

Nevertheless, another study [44] showed that CRP levels decrease following cART treatment, although they are still maintained at high levels. However, these authors related greater levels of CRP to high mortality rates due to cardiovascular alterations after a short period in malnourished HIV-infected adults in Africa, the possible explanation of which was the persistent chronic inflammatory process [44]. Anyway, both levels of CRP as inflammatory cytokines and metabolic profile are related to the lifestyle of individuals. Thus, balanced food intake, poor in sodium, fats, and sugar and with intake of antioxidants, and physical activity, especially a combination of endurance and strength training, are important in maintaining or delaying the onset of lipodystrophy, metabolic syndrome, and other non-AIDS diseases $[45,46]$.

The present research investigated other three poorly studied parameters: AGEs and HMGB1, which are involved in the induction of cellular activation and trigger inflammatory responses, and sRAGE, which plays the opposite effect.

After cART initiation, AGEs plasma levels decreased significantly. Little is known about AGEs concentrations and their effect on the pathogenesis of HIV infection, especially in vivo. To our knowledge, this is the first study to evaluate the effect of cART on the levels of AGEs in asymptomatic PLWHA. However, different reports suggest that their high levels are related to the development of various comorbidities in non-HIV-infected individuals, mainly aging-related complications, such as cataract formation, atherosclerosis [11, 47], osteoporosis [13], Alzheimer's disease [16], chronic kidney disease, and diabetes mellitus [11, 15]. With regard to this, cART seems to contribute to improving this parameter. However, in our study, this improvement was evident only in patients who began cART with lower CD4+ T counts $(<500$ cells $/ \mathrm{mm}^{3}$ ), at least after a short-term period of therapy. Further analyses in long-term cART period are needed to monitor this key parameter in PLWHA.

Differences in sRAGE levels before and after cART introduction were not observed in the present study, possibly because of the short interval analyzed. After all, firstly AGEs activate the inflammatory cascade with consequent production of IL-6, and it is probably only after such induction that sRAGE increase in the circulation occurs [48]. Hence, after cART and with the decrease in AGEs levels, there may have been lower cell stimulation and lower sRAGE production. Another hypothesis is that, even with a slight, nonsignificant posttherapy sRAGE increase, there may have been a larger capture of AGEs by these soluble receptors, thus significantly reducing their circulating levels in HIV patients after cART.

In the general population, alterations in levels of blood have been associated with diseases typical of aging, especially those related to metabolic syndrome [10, 21, 22, 49]. Studies on sRAGE are scarce in the field of HIV/AIDS despite its importance for both virus pathogenesis and the management of chronic diseases in infected individuals. Jeong et al. [21] found that high concentrations of sRAGE were related to lower development of atherosclerosis in PLWHA, suggesting a protective role for this marker in one of the major and most serious comorbidities found in these individuals. Thus, these authors [21] suggested that the measurement of plasma sRAGE could be used in clinical practice in an attempt to predict cardiovascular risk in PLWHA. The same authors also showed no differences between the cART classes used, although most patients with high sRAGE were under NNRTI regimen. Likewise, in the present study, the majority of patients $(83.3 \%)$ were receiving a scheme consisting of NNRTI, which referred to efavirenz. Moreover, we did not find any relation between the cART classes used by our patients and the alterations in the studied biomarkers (data not shown).

High systemic levels of HMGB1 also are found in many chronic proinflammatory conditions, including diabetes mellitus and cancers, as well as in acute conditions, such as sepsis $[17,32,50]$. An interesting finding is that HMGB1 can bind and catalyze the movement of LPS monomers from LPS aggregates thus favoring LPS binding to CD14 which eventually increase TNF- $\alpha$ production, via TLR 4 activation [27]. Also, monocyte stimulation with both LPS and HMGB1 results in an increased production of TNF- $\alpha$ compared to the case with LPS alone [27]. This indicates that HMGB1 may also induce HIV reactivation in PBMC of individuals under cART [51]. However, in this study, no differences were observed in HMGB1 levels before and after cART. In contrast, other studies have shown increased levels of HMGB1 in PLWHA in relation to the uninfected population, mainly in patients with advanced disease $[52,53]$. Association between this marker and the VL of the patients was also observed by Trøseid et al. [53], in addition to the reduction in both of them after two years of cART. This was another aspect not observed in this study, perhaps due to the short period analyzed.

Although the present study has analyzed a small sample and does not present data on the dietary inquiries of patients, which may also influence the levels of AGEs [54] and of some metabolic parameters [46], the study population underwent rigorous exclusion criteria in order to minimize possible confounding effects in the results, such as the presence of comorbidities that knowingly induce and are induced by AGEs, sRAGE, and HMGB1. Another limitation of our study is related to the time after beginning therapy for the second analysis, ranging from 6 to 12 months. Shorter measures may not reflect the steady state level of a given cytokine during cART, for example, especially in those individuals that still show detectable VL values. Therefore, although our aim was to check the marker's changes in the therapeutic beginning, the study of these biomarkers in patients on cART for a long period would also be enlightening. Besides that, the lack of a 
control group can be considered another bias of this study, due to the lack of parameters measures that are not well defined in the literature and which could explain some data founded here.

\section{Conclusion}

Finally, we concluded that, in the first months of cART, there was a decrease in glucose pathway oxidation, reducing AGEs soluble especially in individuals with initial CD4+ $\mathrm{T}<500$ cells $/ \mathrm{mm}^{3}$, which would indicate lower immune activation and an improvement in the inflammatory status of these patients. However, the analysis of other inflammatory markers did not show such benefit since the levels of inflammatory cytokines were not modified and those of CRP increased over the same period. Another negative aspect was the increase in triglycerides in this short period. Because HMGB1 and sRAGE levels were not modulated by initial therapy, other pattern-recognition receptors and other products responsible for cell activation and inflammation could be more informative in the analyses of chronic inflammation in HIV-infected individuals.

Therefore, analysis of other markers and further studies with larger cohorts and in the long term are important to clarify the influence of drug treatment on the mechanisms of activation, inflammation, and metabolic changes that affect the PLWHA. Thence, despite the incontestable benefits of cART in controlling HIV replication, some strategies could be useful in preventing/delaying the onset of non-AIDS-defining illnesses, such as physical exercises practice, a balanced diet, and other healthy lifestyle habits.

\section{Competing Interests}

The authors declare that they have no conflict of interests.

\section{Acknowledgments}

This work was supported by grants from the Brazilian Agency "CAPES" (Coordenação de Aperfeiçoamento de Pessoal de Nível Superior), to Karen Ingrid Tasca, Caio Cavassan de Camargo; from "Reitoria/UNESP, to Juliana Trindade Caleffi; and from FAPESP” (Fundação de Amparo à Pesquisa do Estado de São Paulo) to Mariana Gatto (number 2010/139225). The authors want to acknowledge the efforts of the personnel from EAP/UNESP, especially Professor Dr. José Eduardo Corrente for thr help with the statistical analysis. The authors thank all the people from Tropical Disease Department, Botucatu Blood Center, Laboratory of Tropical Diseases and Experimental Research Unit (FMB/UNESP), especially Professor Dra. Alexandrina Sartori, Professor Dra. Cilmery S. Kurokawa, Maria Regina Moretto, and Silvia R. T. Estevan for all help. The authors also thank the patients and employees of Specialized Outpatient Service of Infectology "Domingos Alves Meira."

\section{References}

[1] UNAIDS.org. Joint United Nations Programme on HIV/AIDS, Global AIDS Response Progress Reporting, 2015, http://www .unaids.org/en/dataanalysis/knowyourresponse/globalaidsprogressreporting/.

[2] C. S. Rodrigues, M. D. Guimarães, F. A. Acurcio, and C. C. Comini, "Interrupção do acompanhamento clínico ambulatorial de pacientes infectados pelo HIV," Revista de Saúde Publica, vol. 37, no. 2, pp. 183-190, 2003.

[3] A. J. Aberg, "Aging, inflammation, and HIV," Topics in Antiviral Medicine, vol. 20, no. 3, pp. 101-105, 2012.

[4] Ministério da Saúde, Departamento de DST, Aids e Hepatites Virais, Boletim Epidemiologia Dst Aids, 2014.

[5] G. Guaraldi, G. Orlando, S. Zona et al., "Premature age-related comorbidities among HIV-infected persons compared with the general population," Clinical Infectious Diseases, vol. 53, no. 11, pp. 1120-1126, 2011.

[6] K. V. Ramana, "Effect of highly active antiretroviral therapy (HAART) on human immunodeficiency virus disease pathogenesis and progression," American Journal of Public Health Reserch, vol. 2, no. 3, pp. 68-74, 2014.

[7] S. G. Deeks, "HIV infection, inflammation, immunosenescence, and aging," Annual Review of Medicine, vol. 62, pp. 141-155, 2011.

[8] K. Venkataramana, "A study of biological markers in HIV disease progression and management in the highly active antiretroviral therapy (HAART) era," American Journal of Bioscience and Bioenginnering, vol. 1, no. 2, pp. 24-37, 2013.

[9] T. Hulgan, J. Morrow, R. T. D’Aquila et al., "Oxidant stress is increased during treatment of human immunodeficiency virus infection," Clinical Infectious Diseases, vol. 37, no. 12, pp. 17111717, 2003.

[10] B. I. Hudson, A. M. Holfmann, L. Bucciarelli et al., "Glycation and diabetes: the RAGE connection," Current Science, vol. 83, no. 12, pp. 1515-1521, 2002.

[11] B. K. Kilhovd, T. J. Berg, K. I. Birkeland, P. Thorsby, and K. F. Hanssen, "Serum levels of advanced glycation end products are increased in patients with type 2 diabetes and coronary heart disease," Diabetes Care, vol. 22, no. 9, pp. 1543-1548, 1999.

[12] B. Knysz, B. Szetela, and A. Gładysz, "Pathogenesis of HIV-1 infection-chosen aspects," HIV \& AIDS Review, vol. 6, no. 1, pp. 7-11, 2007.

[13] P. Pietschmann, D. Mechtcheriakova, A. Meshcheryakova, U. Föger-Samwald, and I. Ellinger, "Immunology of osteoporosis: a mini-review," Gerontology, vol. 62, no. 2, pp. 128-137, 2016.

[14] M. Kaneko, L. Bucciarelli, Y. C. Hwang et al., "Aldose reductase and AGE-RAGE pathways: key players in myocardial ischemic injury," Annals of the New York Academy of Sciences, vol. 1043, pp. 702-709, 2005.

[15] A. G. Huebschmann, J. G. Regensteiner, H. Vlassara, and J. E. B. Reusch, "Diabetes and advanced glycoxidation end products," Diabetes Care, vol. 29, no. 6, pp. 1420-1432, 2006.

[16] V. Srikanth, A. Maczurek, T. Phan et al., "Advanced glycation endproducts and their receptor RAGE in Alzheimer's disease," Neurobiology of Aging, vol. 32, no. 5, pp. 763-777, 2011.

[17] G. P. Sims, D. C. Rowe, S. T. Rietdijk, R. Herbst, and A. J. Coyle, "HMGB1 and RAGE in inflammation and cancer," Annual Review of Immunology, vol. 28, pp. 367-388, 2010.

[18] K. Kierdorf and G. Fritz, "RAGE regulation and signaling in inflammation and beyond," Journal of Leukocyte Biology, vol. 94, no. 1, pp. 55-68, 2013. 
[19] A. Z. Kalea, A. M. Schmidt, and B. I. Hudson, "RAGE: a novel biological and genetic marker for vascular disease," Clinical Science, vol. 116, no. 8, pp. 621-637, 2009.

[20] A. M. Schmidt, S. D. Yan, S. F. Yan, and D. M. Stern, "The multiligand receptor RAGE as a progression factor amplifying immune and inflammatory responses," Journal of Clinical Investigation, vol. 108, no. 7, pp. 949-955, 2001.

[21] S. J. Jeong, C. O. Kim, Y. G. Song et al., "Low plasma levels of the soluble receptor for advanced glycation end products in HIV-infected patients with subclinical carotid atherosclerosis receiving combined antiretroviral therapy," Atherosclerosis, vol. 219, no. 2, pp. 778-783, 2011.

[22] F. Santilli, L. Bucciarelli, D. Noto et al., "Decreased plasma soluble RAGE in patients with hypercholesterolemia: effects of statins," Free Radical Biology \& Medicine, vol. 43, no. 9, pp. 12551262, 2007.

[23] D. Geroldi, C. Falcone, E. Emanuele et al., "Decreased plasma levels of soluble receptor for advanced glycation end-products in patients with essential hypertension," Journal of Hypertension, vol. 23, no. 9, pp. 1725-1729, 2005.

[24] J. O. Thomas, "HMG 1 and 2: architectural DNA-binding proteins," Biochemical Society Transactions, vol. 29, no. 4, pp. 395-401, 2001.

[25] P. Scaffidi, T. Misteli, and M. E. Bianchi, "Release of chromatin protein HMGB1 by necrotic cells triggers inflammation," Nature, vol. 418, pp. 191-195, 2002.

[26] J. Tian, A. M. Avalos, S.-Y. Mao et al., “Toll-like receptor 9dependent activation by DNA-containing immune complexes is mediated by HMGB1 and RAGE," Nature Immunology, vol. 8, no. 5, pp. 487-496, 2007.

[27] J. H. Youn, Y. J. Oh, E. S. Kim, J. E. Choi, and J.-S. Shin, "High mobility group box 1 protein binding to lipopolysaccharide facilitates transfer of lipopolysaccharide to CD14 and enhances lipopolysaccharide-mediated TNF- $\alpha$ production in human monocytes," The Journal of Immunology, vol. 180, no. 7, pp. 5067-5074, 2008.

[28] V. Urbonaviciute, B. G. Fürnrohr, S. Meister et al., "Induction of inflammatory and immune responses by HMGB1-nucleosome complexes: implications for the pathogenesis of SLE," Journal of Experimental Medicine, vol. 205, no. 13, pp. 3007-3018, 2008.

[29] M.-L. Gougeon, M.-T. Melki, and H. Saïdi, "HMGB1, an alarmin promoting HIV dissemination and latency in dendritic cells," Cell Death \& Differentiation, vol. 19, no. 1, pp. 96-106, 2012.

[30] U. Andersson and H. E. Harris, "The role of HMGB1 in the pathogenesis of rheumatic disease," Biochimica et Biophysica Acta-Gene Regulatory Mechanisms, vol. 1799, no. 1-2, pp. 141$148,2010$.

[31] T. Yasuda, T. Ueda, Y. Takeyama et al., "Significant increase of serum high-mobility group box chromosomal protein 1 levels in patients with severe acute pancreatitis," Pancreas, vol. 33, no. 4, pp. 359-363, 2006.

[32] S. Zhang, J. Zhong, P. Yang, F. Gong, and C.-Y. Wang, "HMGB1, an innate alarmin, in the pathogenesis of type 1 diabetes," International Journal of Clinical and Experimental Pathology, vol. 3, no. 1, pp. 24-38, 2010.

[33] D. V. Havlir, I. C. Marschner, M. S. Hirsch et al., "Maintenance antiretroviral therapies in HIV-infected subjects with undetectable plasma HIV RNA after triple-drug therapy," The New England Journal of Medicine, vol. 339, no. 18, pp. 1261-1268, 1998.

[34] A. E. C. G. Fukumoto, C. C. Oliveira, K. I. Tasca, and L. D. R. D. Souza, "Evolution of patients with AIDS after cART: clinical and laboratory evolution of patients with AIDS after 48 weeks of antiretroviral treatment," Revista do Instituto de Medicina Tropical de Sao Paulo, vol. 55, no. 4, pp. 267-273, 2013.

[35] L. F. D. S. Pinto Neto, M. B. das Neves, R. Ribeiro-Rodrigues, K. Page, and A. E. Miranda, "Dyslipidemia and fasting glucose impairment among HIV patients three years after the first antiretroviral regimen in a Brazilian AIDS outpatient clinic," Brazilian Journal of Infectious Diseases, vol. 17, no. 4, pp. 438443, 2013.

[36] S. J. Souza, L. A. Luzia, S. Santos, and P. H. C. Rondó, "Lipid profile of HIV-infected patients in relation to antiretroviral therapy: a review," Revista da Associação Médica Brasileira, vol. 59, no. 2, pp. 186-198, 2013.

[37] J. M. Brenchley, D. A. Price, T. W. Schacker et al., "Microbial translocation is a cause of systemic immune activation in chronic HIV infection," Nature Medicine, vol. 12, no. 12, pp. 1365-1371, 2006.

[38] D. C. Hsu, I. Sereti, and J. Ananworanich, "Serious Non-AIDS events: immunopathogenesis and interventional strategies," AIDS Research and Therapy, vol. 10, no. 1, article 29, 2013.

[39] P. W. Hunt, "HIV and inflammation: mechanisms and consequences," Current HIV/AIDS Reports, vol. 9, no. 2, pp. 139-147, 2012.

[40] J. L. Fahey, "Plasma immune activation markers, and clinically relevant surrogate markers in human immunodeficiency virus infection," Cytokines, vol. 5, no. 5, pp. 597-603, 1998.

[41] S. Hattab, A. Guihot, M. Guiguet et al., "Comparative impact of antiretroviral drugs on markers of inflammation and immune activation during the first two years of effective therapy for HIV1 infection: An Observational Study," BMC Infectious Diseases, vol. 14, no. 1, article 122, 2014.

[42] M. Masiá, E. Bernal, S. Padilla et al., "The role of C-reactive protein as a marker for cardiovascular risk associated with antiretroviral therapy in HIV-infected patients," Atherosclerosis, vol. 195, no. 1, pp. 167-171, 2007.

[43] M. M. M. Guimarães, D. B. Greco, S. M. D. Figueiredo, R. B. Fóscolo, A. R. D. Oliveira Jr., and L. J. D. C. Machado, "Highsensitivity C-reactive protein levels in HIV-infected patients treated or not with antiretroviral drugs and their correlation with factors related to cardiovascular risk and HIV infection," Atherosclerosis, vol. 201, no. 2, pp. 434-439, 2008.

[44] M. Bestawros, T. Chidumayo, M. Blevins et al., "Increased systemic inflammation is associated with cardiac and vascular dysfunction over the first 12 weeks of antiretroviral therapy among undernourished, HIV-infected adults in Southern Africa," Journal of AIDS and Clinical Research, vol. 6, no. 3, p. 431, 2015.

[45] B. Lindegaard, T. Hansen, T. Hvid et al., "The effect of strength and endurance training on insulin sensitivity and fat distribution in human immunodeficiency virus-infected patients with lipodystrophy," The Journal of Clinical Endocrinology \& Metabolism, vol. 93, no. 10, pp. 3860-3869, 2008.

[46] L. A. Willig and E. T. Overton, "Metabolic complications and glucose metabolism in HIV infection: a review of the evidence," Current HIV/AIDS Report, vol. 13, pp. 289-296, 2016.

[47] R. D. Semba, E. J. Nicklett, and L. Ferrucci, "Does accumulation of advanced glycation end products contribute to the aging phenotype?" Journals of Gerontology-Series A, vol. 65, no. 9, pp. 963-975, 2010.

[48] A. I. Serban, L. Stanca, O. I. Geicu, and A. Dinischiotu, "Ages-induced IL-6 synthesis precedes RAGE up-regulation 
in HEK 293 cells: an alternative inflammatory mechanism?" International Journal of Molecular Sciences, vol. 16, no. 9, pp. 20100-20117, 2015.

[49] B. I. Hudson, C. Dong, H. Gardener et al., "Serum levels of soluble receptor for advanced glycation end-products and metabolic syndrome: the Northern Manhattan Study," Metabolism: Clinical and Experimental, vol. 63, no. 9, pp. 1125-1130, 2014.

[50] H. Wang, O. Bloom, M. Zhang et al., "HMG-1 as a late mediator of endotoxin lethality in mice," Science, vol. 285, no. 5425, pp. 248-251, 1999.

[51] S. Thierry, J. Gozlan, A. Jaulmes et al., "High-mobility group box 1 protein induces HIV-1 expression from persistently infected cells," AIDS, vol. 21, no. 3, pp. 283-292, 2007.

[52] P. Nowak, B. Barqasho, and A. Sönnerborg, "Elevated plasma levels of high mobility group box protein 1 in patients with HIV1 infection," AIDS, vol. 21, no. 7, pp. 869-871, 2007.

[53] M. Trøseid, A. Sönnerborg, and P. Nowak, "High mobility group box protein-1 in HIV-1 infection: connecting microbial translocation, cell death and immune activation," Current HIV Research, vol. 9, no. 1, pp. 6-10, 2011.

[54] J. Uribarri, M. D. del Castillo, M. P. de la Maza et al., "Dietary advanced glycation end products and their role in health and disease," Advances in Nutrition, vol. 6, no. 4, pp. 461-473, 2015. 


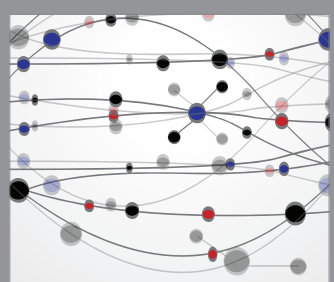

The Scientific World Journal
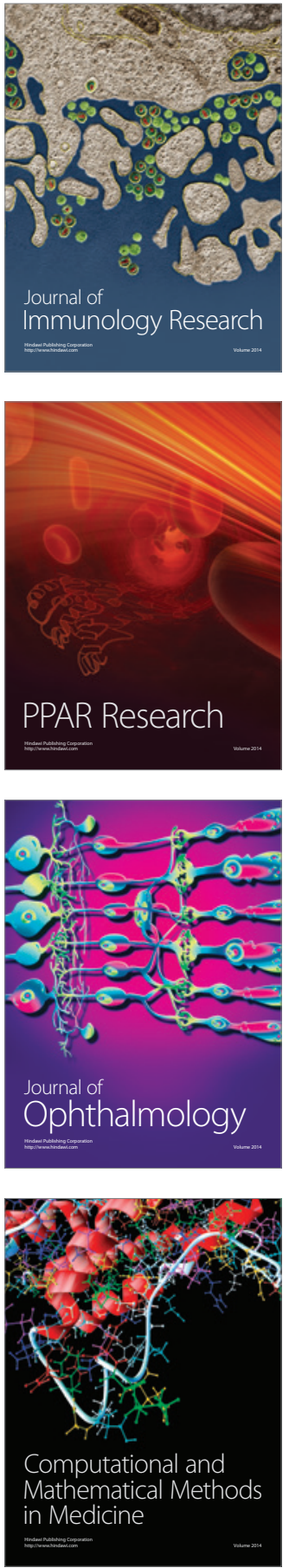

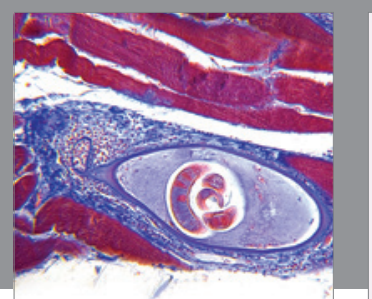

Gastroenterology Research and Practice

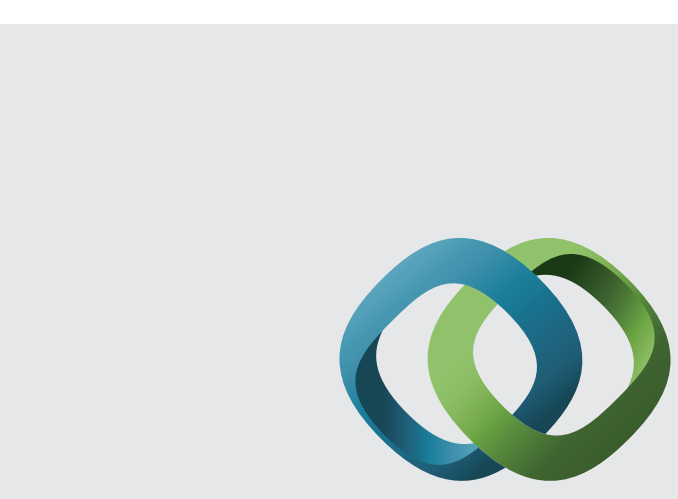

\section{Hindawi}

Submit your manuscripts at

http://www.hindawi.com
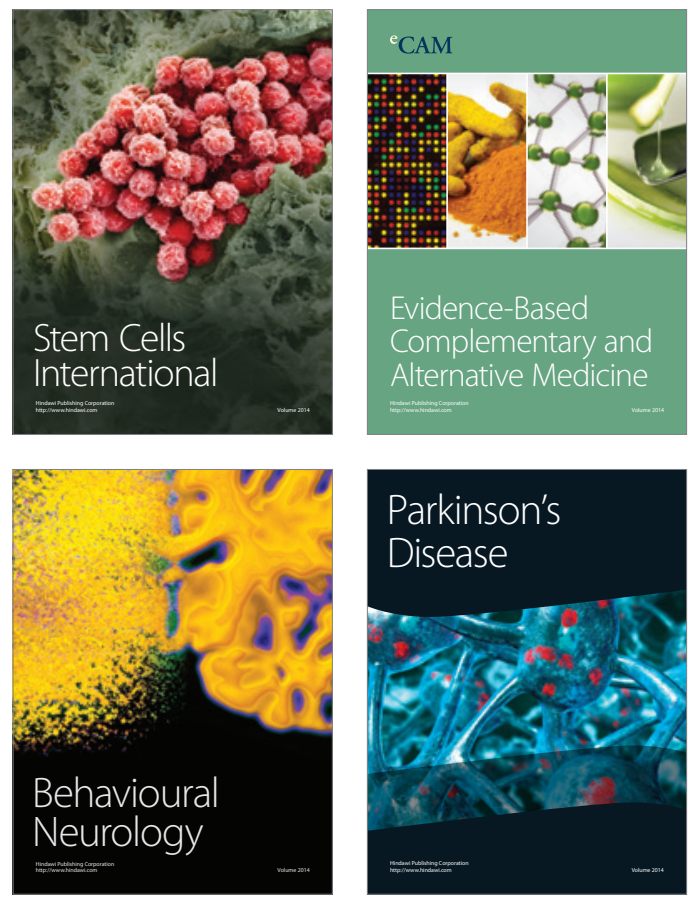
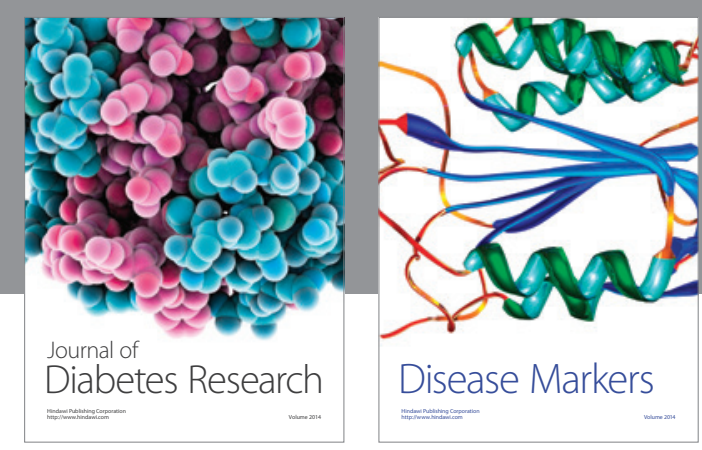

Disease Markers
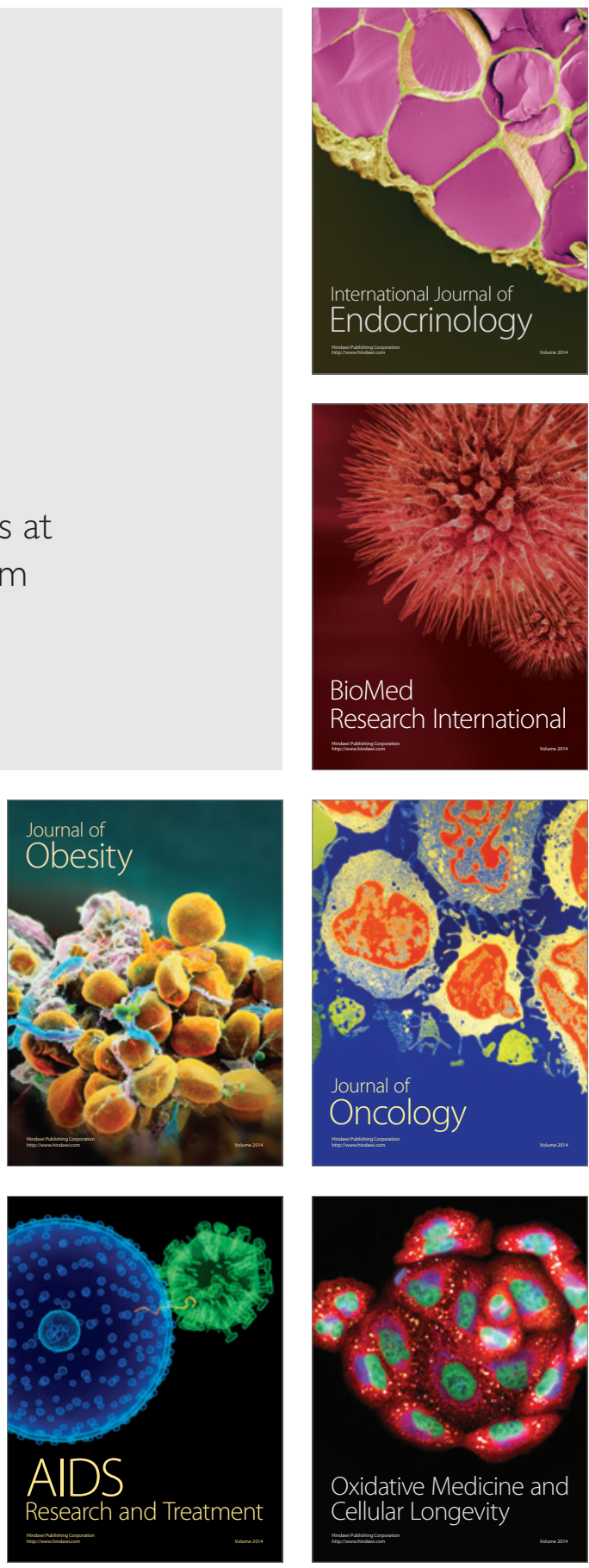